\title{
Commentary to the article: "Rivaroxaban in secondary cardiogenic stroke prevention: two-year single-centre experience based on follow-up of 209 patients"
}

\author{
published in "Kardiologia Polska" 2016; 74, 5: 418-424
}

\author{
Anetta Undas \\ Institute of Cardiology, Jagiellonian University Medical College, Krakow, Poland
}

It is known that a history of stroke or transient ischaemic attack (TIA) is a major risk factor for recurrent ischaemic events as well as bleeding in atrial fibrillation (AF), so effective anticoagulation is of key importance in this high-risk subgroup. Therefore, I read with interest the paper by Lasek-Bal et al. [1]. The authors presented an observational study on Polish patients with AF and previous stroke or TIA, who were treated with rivaroxaban and followed-up for 24 months. The results support the use of rivaroxaban for prevention of recurrent stroke in patients with AF. There are still few studies confronting the current data obtained in Polish clinics with a rapidly growing number of international registries or those from Western European countries. A subgroup analysis of the ROCKET-AF study demonstrated that the efficacy and safety of rivaroxaban compared with warfarin did not differ among AF patients with or without previous stroke or TIA [2]. Unfortunately, the current study did not include a prospective or historic control group on vitamin $\mathrm{K}$ antagonists. It remains to established whether also in Poland rivaroxaban versus warfarin or acenocoumarol are similarly effective and safe when administered to real-life patients with nonvalvular AF.

The authors focused on bleeding complications during follow-up and showed a rather low rate of these events (less than $1 \%, \mathrm{n}=2$ ). Piccini et al. [3] reported in the ROCKET-AF study (with as many as $52 \%$ of AF patients following cerebrovascular events) that during a median follow-up of 1.9 years $5.5 \%$ of patients experienced major bleeding. Surprisingly, epistaxis was common in this group $(6.2 \%)$, while in the ROCKET-AF group $3.2 \%$ of the patients on rivaroxaban experienced this complication in contrast to about $50 \%$ of the bleeding patients who had upper or lower gastrointestinal tract bleeding (one case of a total of 25 in the present study) [3]. The odd pattern of the location of bleeds suggests that in a small cohort the incidence of bleeding events is barely predictable with the single benefit of paramount importance: intracranial bleedings are quite uncommon. For this reason, since none of the patients was lost to follow-up, it would also be of interest to show the characteristics of bleeding AF patients, their renal function in particular. Were there any differences in bleeding risk factors listed in the HAS-BLED scale or other independent risk factors for major bleeding already identified in the ROCKET-AF, namely a history of chronic obstructive pulmonary disease, increased age, baseline diastolic blood pressure $\geq 90 \mathrm{~mm} \mathrm{Hg}$, prior gastrointestinal bleeding, prior acetylsalicylic acid use, or anaemia [4]? Were all the 25 bleeding patients in the high-risk group based on the HAS-BLED score, i.e. with three points or more? Moreover, given the slim chance of approving an antidote to factor Xa inhibitors (e.g. andexanet alpha currently tested in a phase III trial) in the near future, a practising Polish physician dealing with patients receiving rivaroxaban would appreciate information on the management of major bleedings in the patient group analysed by Lasek-Bal et al. [1]. Did the patients receive prothrombin complex concentrates, platelet concentrates, antifibrinolytics, or recombinant factor VIla? Detailed information at least for the two patients who experienced major bleeding, including one case of intracranial bleed, could help optimise the management of rare bleeding events in patients on rivaroxaban in Polish hospitals.

To increase the safety of rivaroxaban use, laboratory monitoring of its anticoagulant effects could be considered. Poor adherence to this agent with a half-life of $8-12 \mathrm{~h}$ is a challenge and inter-individual differences in the anti-Xa activity at the similar time since the last intake of rivaroxaban

\footnotetext{
Address for correspondence:

Anetta Undas, MD, PhD, Institute of Cardiology, Jagiellonian University Medical College, ul. Prądnicka 80, 31-202 Kraków, Poland, tel: +48 1261430 04, fax: +48 1242339 00, e-mail: mmundas@cyf-kr.edu.pl

Kardiologia Polska Copyright (c) Polskie Towarzystwo Kardiologiczne 2016
} 
in subjects with normal renal function has been reported [5]. Moreover, anti-Xa activity is useful to confirm that a bleeding patient has a detectable rivaroxaban concentration in the circulation, or when it is unclear when the patients with bleeding events have taken the last dose of the anticoagulant agent [6]. At least minor bleeding events on treatment do not correlate well with rivaroxaban concentrations [5]. A role of laboratory monitoring in a real-life setting of subjects taking rivaroxaban remains unclear.

It is advisable to run registries for treatment with rivaroxaban in large clinical centres, to assess the impact of local factors known from everyday practice, for instance reduction of daily dose or its intake every other day, due to high cost of the treatment, which is not reimbursed in Poland. Increasing use of rivaroxaban, like other non-vitamin K oral anticoagulants in Poland, in which no specialised anticoagulation clinics were ever organised by our healthcare system in the whole country, is likely to lower the incidence of dangerous bleeding and thromboembolic complications of long-term anticoagulation in Polish patients with $\mathrm{AF}$, and thus good long-term outcomes of everyday stroke prevention requires collection of data on anticoagulated patients in each outpatient clinic, including the benefits and adverse events of the therapy as presented by Lasek-Bal et al. [1]. Initiatives similar to that of Dr Lasek-Bal should be strongly encouraged.
Conflict of interest: Honoraria for lectures received from Bayer, a manufacturer of Xarelto (rivaroxaban).

\section{References}

1. Lasek-Bal A, Urbanek T, Puz P, Piekarski M. Rivaroxaban in secondary cardiogenic stroke prevention: two-year single-centre experience based on follow-up of 209 patients. Kardiol Pol, 2016; 74: 418-424. doi: 10.5603/KP.a2015.0207.

2. Hankey GJ, Patel MR, Stevens SR et al. Rivaroxaban compared with warfarin in patients with atrial fibrillation and previous stroke or TIA: a subgroup analysis of ROCKET AF. Lancet Neurol, 2012; 11: 315-322.

3. Piccini JP, Garg J, Patel MR et al.; ROCKET AF Investigators. Management of major bleeding events in patients treated with rivaroxaban vs. warfarin: results from the ROCKET AF trial. Eur Heart J, 2014; 35: 1873-1880.

4. Goodman SG, Wojdyla DM, Piccini JP et al. Factors associated with major bleeding events: insights from the ROCKET AF trial (rivaroxaban once-daily oral direct factor Xa inhibition compared with vitamin $\mathrm{K}$ antagonism for prevention of stroke and embolism trial in atrial fibrillation). J Am Coll Cardiol, 2014; 63: 891-900.

5. Zalewski J, Rychlak R, Góralczyk T, Undas A, Rivaroxaban concentration in patients with deep vein thrombosis who reported thrombus progression or minor hemorrhagic complications: first Polish experience. Pol Arch Med Wewn, 2014; 124: 553-555.

6. Undas A, Pasierski T, Windyga J, Crowther M. Practical aspects of new oral anticoagulant use in atrial fibrillation. Pol Arch Med Wewn, 2014; 124: 124-135. 